\title{
Research on embedded GPS/GPRS intelligent building security technology
}

\author{
Di Juxing \\ Department of Computer Science \\ Hebei Institute of Architecture Civil Engineering \\ Zhangjiakou, China \\ djxxing@sohu.com
}

\author{
Zhao Jianguang, Si Yachao, fanjingjing \\ Department of Computer Science \\ Hebei Institute of Architecture Civil Engineering \\ Zhangjiakou, China \\ zlong19801016@163.com
}

\begin{abstract}
This paper studied GPS location information of the collection, processing, GPRS data transmission network, intelligent building security system features. Based on this techniques, we designed an embedded GPS/GPRS intelligent building security system, which is composed of ARM9 microprocessor S3C2410, GPS/GPRS module and some peripheral devices. The system is running under Linux OS. The characteristics of the system are flexible, powerful, and high practicality.
\end{abstract} GPRS

Keywords-Embedded system; intelligence building; GPS;

\section{INTRODUCTION}

With the development of the" $A+4 C$ ”technology that is: modern architecture technology (Architecture), modern control technology (Control), Computer Technology (Computer), communication technology (Communication), Cathode Ray Tube image display technology (CRT), intelligent building is developing to integration, a new form of building intelligent systems was formed in some modern buildings, such as intelligent building security systems. In particular, satellite positioning technology (GPS), modern communications technology, ARM microprocessor and embedded operating system development matures, making intelligent building security system more secure, efficient, convenient and flexible.

\section{RESEARCH ON KEY TECHNOLOGIES OF EMBEDDED INTELLIGENT BUILDING SECURITY SYSTEM}

\section{A. GPS location information acquisition and processing}

GPS its full name in English is "Navigation Satellite Timing And Ranging/Global Position System”, means "Navigation satellite timing and ranging/global positioning system” ,abbreviated as NAVSTAR/GPS, called GPS system. The basic principle of GPS positioning is based on instantaneous high-speed movement of the satellite location as the initial data are known, and then uses the method of space from the rear of the intersection to determine the location of the point under test. Suppose time $t(t$ is the unknown ground point under test clock) there is an unknown point(coordinates $\mathrm{x}, \mathrm{y}, \mathrm{z}$ ) on the ground, can be "observed" at least four satellites, such as $\mathrm{A}(\mathrm{x} 1, \mathrm{y} 1, \mathrm{z} 1), \mathrm{B}(\mathrm{x} 2, \mathrm{y} 2, \mathrm{z} 2), \mathrm{C}(\mathrm{x} 3, \mathrm{y3}, \mathrm{z} 3), \mathrm{D}(\mathrm{x} 4, \mathrm{y} 4, \mathrm{z} 4)$, the satellite coordinates obtained from satellite navigation message. We can receive the satellite clock $t 1$ t2、t3、t4; d1 $d 2 、 d 3 、 d 4$ is the distance from satellite $A, B, C, D$ to the ground $(\mathrm{x}, \mathrm{y}, \mathrm{z})$; $\mathrm{c}$ is the speed of light, so that can be identified the following four equations:

$$
\begin{aligned}
& {\left[\left(x_{1}-x\right)^{2}+\left(y_{1}-y\right)^{2}+\left(z_{1}-z\right)^{2}\right\rfloor+c\left(t_{1}-t\right)=d_{1}} \\
& {\left[\left(x_{2}-x\right)^{2}+\left(y_{2}-y\right)^{2}+\left(z_{2}-z\right)^{2}\right\rfloor+c\left(t_{2}-t\right)=d_{2}} \\
& {\left[\left(x_{3}-x\right)^{2}+\left(y_{3}-y\right)^{2}+\left(z_{3}-z\right)^{2}\right\rfloor+c\left(t_{3}-t\right)=d_{3}} \\
& \left\lfloor\left(x_{4}-x\right)^{2}+\left(y_{4}-y\right)^{2}+\left(z_{4}-z\right)^{2}\right\rfloor+c\left(t_{4}-t\right)=d_{4}
\end{aligned}
$$

GPS module receives GPS users through the satellite signal, after processing the user position, velocity and time information. The monitoring system uses the GPS module uses the communication protocol NMEA0183, provides a serial communication interface, serial communication parameters set as follows: baud rate $=4800 \mathrm{~b} / \mathrm{s}$; data bit 8 ; stop bits $=1$; no parity. Per second through a serial communication interface terminal to the monitoring data processing data transfer 7. Practical positioning applications, the only required periodically to read one of the useful data, such as latitude, longitude and time data, and the remaining information can be ignored. NMEA0183 protocol example:

\$ GPRMC, $<1>,<2>,<3>,<4>,<5>,<6>,<7>,<8>$, $<9>,<10>,<11>,<12>*$ hh $<\mathrm{CR}>,<\mathrm{LF}>$

$\$$ : Information marked the beginning

GPRMC: Recommended positioning information

$<1>$ : UTC time, hhmmss (when minutes and seconds) format

$<2>$ : location state, $\mathrm{A}=$ effective positioning, $\mathrm{V}=$ invalid positioning

$<3>$ : latitude, ddmm.mmmm (degrees minutes) format (in front of 0 will be transmitted)

$<4>$ : Latitude hemisphere $\mathrm{N}$ (northern) or S (southern)

$<5>$ : longitude, dddmm.mmmm (degrees minutes) format (in front of 0 will be transmitted)

$<6>$ : Longitude hemisphere, E (E) or W (W)

$<7>$ : Ground rate $(000.0 \sim 999.9$, in front of 0 will be transmitted)

$<8>$ : Surface course $(000.0 \sim 359.9$ degrees to true north as the reference standard, the front will also be transmitted 0 ) $<9>$ : UTC date, ddmmyy (moon of) format

$<10$ ": magnetic declination, (000.0 to 180.0 degrees, the front will also be transmitted 0 )

$<11$ ": the direction of the magnetic declination, $\mathrm{E}$ (East) or W (West)

$<12$ ": mode instructions (only NMEA0183 3.00 version of the output, $\mathrm{A}=$ autonomous positioning, $\mathrm{D}=$ check points, $\mathrm{E}=$ Estimated, $\mathrm{N}=$ data not valid) 
*: Checksum delimiter

hh: Checksum

[CR] [LF]: carriage return and line termination of the information used in combination.

As long as the GPS module is active, it will continue to NMEA0183 format via the serial port for GPS navigation and positioning information. These data must be fixed format each field through the program information from the cache to take out the byte stream, convert it into a meaningful, the availability of location information data, and then through GPRS module handler to process and send out In order to achieve the positioning of intelligent building security systems and alarms.

\section{B. GPRS data transmission network}

General Packet Radio Service GPRS (General Packet Radio Service) is the use of the concept of the development of packet switching a wireless transmission. GPRS network is based on the existing GSM (Global System for Mobile Telecommunications System) networks, in existing GSM networks need to increase the number of nodes, such as the GGSN (GPRS Gateway Support Node) and SGSN (GPRS Support Node). One main function of SGSN is to record the current location information of mobile terminals and mobile terminals and the GGSN in the mobile packet data between the completion of the sending and receiving. The system uses the GPRS module is designed by the French WAVECOM Q2403A-type modules, dual-band $(900 / 1800 \mathrm{MHz})$, supporting the general AT commands to complete basic mobile data services such as call and receive, send and receive short messages and so on. The wireless network module will respond to commands received back the results to the serial code.

- $\quad$ access to the Internet through GPRS DTU

GPRS DTU GSM networks first landing, then GPRS PPP dial-up, dial-up is successful, GPRS DTU will be randomly assigned by a moving internal IP address (usually 10.XXX), and its internal network IP addresses are usually not fixed, Change with each dial.

GPRS DTU initiate communication with the data center connectivity, and maintain communication links have been there. Data center or fixed public network IP address domain name as a parameter stored in the GPRS DTU, the power to dial-up GPRS DTU once successful, can take the initiative to connect to the data center.

GPRS DTU through the data center IP address (if using central domain, then, first through the center of domain name resolution the center IP address) and port number and other parameters to the data center initiated TCP or UDP traffic requests, by the center response after, GPRS DTU shake hands with the Centre is that successful, and then to maintain this communication link has always existed, if the communication connection is lost, GPRS DTU and the center will immediately re-handshake until the TCP/UDP communications connection is established, the data can be carried out two-way communications.

Must be lawful and valid SIM card, the system immediately after the successful launch of the module through UART port to read and write operations. Because the module is running in the embedded Linux platform, it needs to start a terminal window, start Minicom for serial operation.

- $\quad$ Send and receive text messages through GPRS to achieve security alarm module

When an alarm signal, ARM S3C2410 immediately start GPRS module connected to the network through the GPRS DTU, and send a SMS alarm message to the alarm center, enabling the wireless alarm. SMS AT control commands sent by the following:

(1) Select the short message mode

AT + CMGF: priority information format. TEXT execution mode and PDU format mode. Such as: AT + CMGF $=1$ is used to select the short message format, a format for text, 0 for PDU format, return "OK".

(2) send a message

Enter AT $+\mathrm{CMGF}=1$ (send in text format, such as the use PDU format, the AT + CMGF $=0$ )

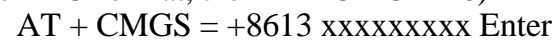

$>$ short message content. Crtl $+\mathrm{Z}$ and send the end, the successful return OK.

(2) receive information

AT + CPMS: priority information storage. Information is used to read and write the definition of the storage area.

AT + CNMI: New information tips. Choose how to receive text messages from the network.

$\mathrm{AT}+\mathrm{CMGR}$ : Reading SMS. From the + CPMS command to set the memory read.

AT + CMGD: deleted text messages.

\section{The design principles of intelligent building security} system

Security system in the intelligent building design, you should follow the following principles:

- Reliability: The reliability of the security system is the first. Both the system design, equipment selection, installation, commissioning should be based on reliability as a precondition.

- Independence: Security system should be independent for the integrated management system, and must not be weak as other subsystems of the hybrid system management, to reduce the variety of security systems may have been damaged or disturbed.

- Security: Security System program have the ability to prevent unauthorized use, access, or destruction.

- Linkage: security system linkage with other systems, such as fire systems, lighting systems, and for other system services.

- $\quad$ scalability, ease, practicality, economy and so on.

\section{DESIGN AND IMPLEMENTATION OF INTELLIGENT SECURITY SYSTEM}

When the ARM S3C2410 received alarm sensor alarm signal to start the GPS and read useful information to determine the location and time, and immediately start the GPRS system server to the monitoring center and the owners mobile terminal to send alarm information. Monitoring 
center can send SMS commands to take the necessary measures. Intelligent building security system structure shown in Figure 1.

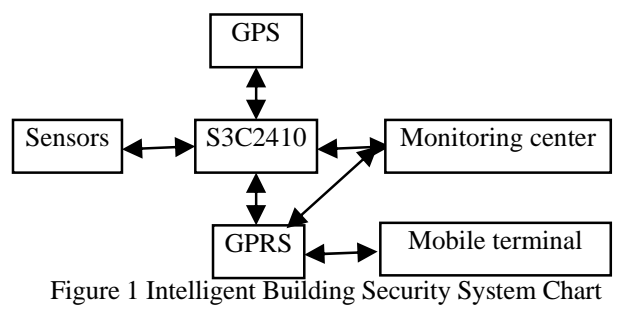

\section{A. Hardware Design}

The system uses Samsung's S3C2410A microprocessor control chip as the detection side. As a modern intelligent building security system widely used in the ARM9 chip, S3C2410A 16/32-bit RISC is a microprocessor, using the ARM920T core, provides the following extensive internal equipment, handheld devices and general types of applications a low price , Low power, high-performance small-scale micro-controller solution. By providing a full range of system peripherals, S3C2410A greatly reduce the cost of the entire system, the system eliminates the need to configure additional components.

Security test terminal shown in Figure 2, S3C2410A as the detection. The core section includes GPRS control, GPS control, all kinds of alarm sensor control and the external interface. CPU embedded PPP and TCP/IP protocol stack, but were controlled through a serial port UART0 GPRS to work, through serial port UART1 control the operation of GPS.
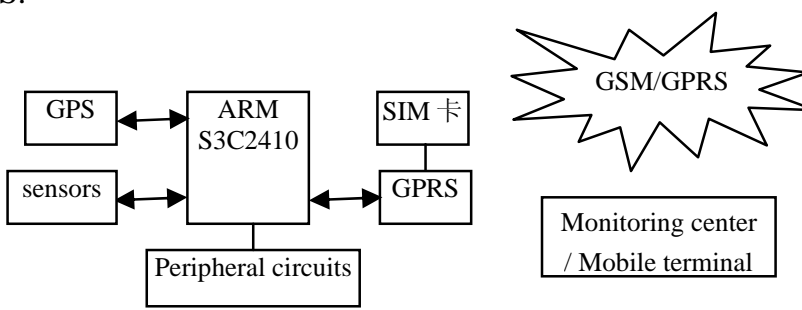

Figure 2 Intelligent Building Security Monitoring terminal

- Of which: ARM control of GPRS is mainly through the serial port UART0 to send AT command. In the system design, CPU can control the I/O ports highlow, to achieve the GPRS reset, the main program once the run, not short for GPRS module detection, abnormality was found immediately by controlling the I/O port Level to achieve reset.

- ARM, mainly through the control of the GPS serial port UART1 send control commands to achieve (GPS sampling period settings, GPS output data selection, communication baud rate settings, etc.). Meanwhile, the GPS receiver through the serial port UART1 data.

- ARM on the other peripheral interface control switch mainly through GPIO interface input/output, S3C2410A offers 117 Road GPIO interface, may need access to a variety of sensors (Tamper pressure sensor, fire sensor, infrared shooting Sensors, etc.).
- ARM overall control of the system: First, ARM completed its GPS, GPRS and peripheral interface circuits of the initialization, and to detect the status of various security sensors (infrared radiation sensing, pressure sensing tamper, etc.); Second, ARM GPIO input continuously monitor the state, when the alarm signal input, start GPS module to begin receiving location information, and within this information is stored in flash; UART0 serial port to send AT commands to start sending messages, and GPS receiver The data sent via GPRS to the alarm host and the mobile terminal.

\section{B. System Software Design}

In the whole security system, involving the monitoring center server software, security test side host, the underlying software. Then this describes only the host-side security testing software design.

In the GPS/GPRS Intelligent Building Security Systems security detection side software design, using Linux, C language design and modular design, software consists of a main program and subroutine modules. Among them, the routine completion of some single function, main program module to complete all routine scheduling and coordination of the block diagram shown in Figure 3.

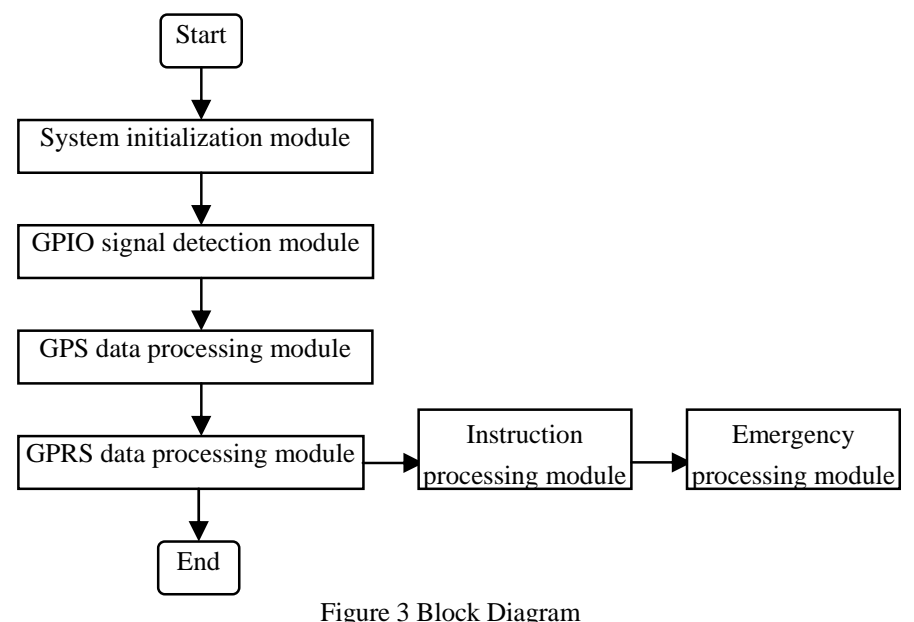

The following design features to make the following main program explained: When the system is initialized later, into the GPIO module, this module is mainly read from the $\mathrm{I} / \mathrm{O}$ port level signal, the alarm signal is input when the $\mathrm{I} / \mathrm{O}$ port will change the level, CPU level change is detected after the data processing module into the GPS, if not detected changes in the circulating level of detection, until the detected level changes; CPU into the GPS signal is detected then the data Processing module, first set the baud rate and other parameters such as GPS, and then receive satellite data, receiving data transmitted through the serial port to the CPU, and stored in flash, the completion of storage, start the GPRS module. In this module, first read the data stored in the satellite, sending AT command to start sending messages, after the successful launch of satellite data as the information content to be sent, to send, send a successful return, and to 
detect whether there is new information received, If reading the instructions, CPU reads the instruction is received into the order processing module of instruction, to take corresponding measures according to the instruction sheet.

Monitoring center or the mobile terminal receives the alarm information, you can send the appropriate emergency treatment instructions, send the command will be sent to the GPRS module to read and ARM, and then take corresponding measures according to the instruction sheet.

Which, with the data exchange, through the serial port UART0 interrupt to complete; and GPS data exchange, via the serial port UART1 completed. They are in the form of subroutines and main program, but also including some of the GPS, GPRS module initialization and setup, control command parsing, and PPP protocols, TCP/IP protocol analysis and implementation subroutine.

\section{CONCLUSION}

According to the characteristics of embedded systems and intelligent building security system requirements, based on the ARM2410 and embedded Linux platform to develop a set of embedded GPS/GPRS intelligent building security systems. After repeated testing system is stable, works well, the system is extremely broad market prospect.

\section{REFERENCES}

[1] Yang Hui, Hu Jian, Lin Zhiquan. S3C2410-based embedded Linux and image transmission system [J]. Computer, 2007 (3) :20-24.

[2] Xu Jing. embedded system design and development examples Detailed - ARM-based applications [M]. Beijing: Beijing Aerospace University Press, 2005.

[3] Li Yunfeng and so on. S3C2410-based embedded system applications in automatic meter reading in the [J]. Electrical Measurement and Instrumentation, 2007 (3): 22 - 27.

[4] Cai Ning fruit, He Xiaoqiong. 8-bit MCU with Ethernet serial converter [J]. Electronic Technology Application, 2002 (2) :52-55.

[5] Wang Tianmiao. Embedded System Design and Case Development [M]. Beijing: Tsinghua University Press, 2003.

[6] Li Shanping, Liu bee. Linux kernel 2.4 source code analysis Daquan [M]. Beijing: Mechanical Industry Press, 2002.

[7] Wang Jian and so on. DSP and ARM-based embedded multimedia terminal design and implementation [J]. Electronic Measurement Technology, 2006 -29-2:56-57

[8] Wang Xingjie and so on. Embedded Linux-based cross-development techniques [J]. Computer Applications 2008-25-1:206-208

[9] Li Feng and so on. Embedded Linux kernel and the process of tailoring the specific method of [J]. IT 2009-33-5:223-225

[10] Sun Tianze, Yuan Wenju. Embedded Linux driver development guide the design and ARM9-based processor [M]. Beijing: Electronic Industry Press,2007,133-135 